\title{
Impatiens bijieensis (Balsaminaceae), a new species from karst plateau in Guizhou, China
}

\author{
Liu-Yi Ren', Yi Chen', Tao-Hua Yuan', \\ Rong-Xin Huang', Mei-Jun Li', Xin-Xiang Bai' \\ I College of Forestry, Guizhou University CN-550025 Guiyang, China \\ Corresponding author: Xin-Xiang Bai (254715174@qq.com)
}

Academic editor: L.J. Dorr | Received 3 November 2021 | Accepted 17 February 2022 | Published 4 March 2022

Citation: Ren L-Y, Chen Y, Yuan T-H, Huang R-X, Li M-J, Bai X-X (2022) Impatiens bijieensis (Balsaminaceae), a new species from karst plateau in Guizhou, China. PhytoKeys 192: 1-10. https://doi.org/10.3897/phytokeys.192.77517

\begin{abstract}
Impatiens bijieensis X.X. Bai \& L.Y. Ren, sp. nov. from northwest Guizhou Province, China, is described and illustrated. This new species is distributed discontinuously in Jiulongshan, Dafang County and Dajiucaiping, Hezhang County, both of which are in the Wumeng Mountain area, a karst plateau landform. The new species is morphologically similar to I. pterosepala Hook.f., I. lasiophyton Hook.f. and I. leptocaulon Hook.f. in height and flower shape and it especially resembles I. lasiophyton in pilosity. However, it differs in its deep purplish-red flower, 2-lobed lower sepal apex and cylindrical capsule. A detailed description, colour photographs and a provisional IUCN Red List assessment are provided along with discussions of its geographical distribution, ecology and morphological relationships with other similar species.
\end{abstract}

\section{Keywords}

Balsaminaceae, China, Impatiens, morphology, new species

\section{Introduction}

The genus Impatiens Linnaeus (1753: 937), belonging to the Balsaminaceae, consists of more than 1000 species, mainly distributed in the montane forests of the tropics and subtropics of the Old World with five centres of diversity, namely tropical Africa, Madagascar, south India and Sri Lanka, eastern Himalaya and Southeast Asia (Grey- 
Wilson 1980; Song et al. 2003; Yuan et al. 2004; Mabberley 2017). In recent years, a few new species have also been found in the northern temperate regions of Europe, Russia and China, as well as North America (Liao et al. 2021). Currently, there are more than 349 species of Impatiens in China (Yuan et al., in press) which are distributed mainly in the southwest and northwest mountainous regions, especially in southwest Provinces (including Guizhou, Yunnan, Sichuan) (Chen 2001; Chen et al. 2007; Cai et al. 2015; Kuang 2015; Tan et al. 2015; Ding et al. 2016, 2017; Xia et al. 2019; Gu et al. 2021; Liao et al. 2021; Peng et al. 2021b; Song et al. 2021a, b, c) and 61 species of Impatiens have been reported in Guizhou Province (Xiong and Yang 2009; Cong 2010; Kuang et al. 2014; Luo and Deng 2015; Peng et al. 2021a; Yu et al. 2021; Yuan et al. in press).

Impatiens are morphologically characterised by their petals always united in pairs into lateral, united petals; fruit a fleshy, explosive capsule; seeds often dispersed elastically from valves when ripe (Chen et al. 2007). From September 2014 to October 2021, during our field investigation in Bijie City, northwest Guizhou Province, China, we encountered an unknown Impatiens species. The plants were found growing in gullies between gently sloping mountain meadows of karst plateau, this special habitat distinguishing our plants from other known species. After a thorough morphological study, based on literature (Xiong and Luo 1989; Chen 2001; Chen et al. 2007; Yu 2012) and herbarium material (GZAC!), we concluded that this Impatiens species should be placed in I. subg. Impatiens as it differed from previously reported or described taxa and we describe it here as a new species.

\section{Materials and methods}

The material for this study was mainly collected from the survey of wild ornamental plant resources in Guizhou Province, China. The morphological description of the new species was based on careful examination of fresh material in the field and herbarium specimens. Comparisons with other species were made to virtual herbarium specimens (GZAC, HC, HIB, IBK, IBSC and PE), photographs and literature (Hooker 1908a; Grey-Wilson 1980; Chen 2001; Chen et al. 2007; Yu 2012).

\section{Taxonomic treatment}

Impatiens bijieensis X.X. Bai \& L.Y. Ren, sp. nov. urn:lsid:ipni.org:names:77265014-1

Figs 1, 2, 3A-C

Type. China. Guizhou: Hezhang County, Xingfa Town, Dajiucaiping, $2763 \mathrm{~m}$ alt., 2959'53"N, 10445'29"E, 20 Aug 2021, X.X. Bai \& L.Y. Ren DJCP 20210820 (holotype: GZAC!; isotype: PE!). 

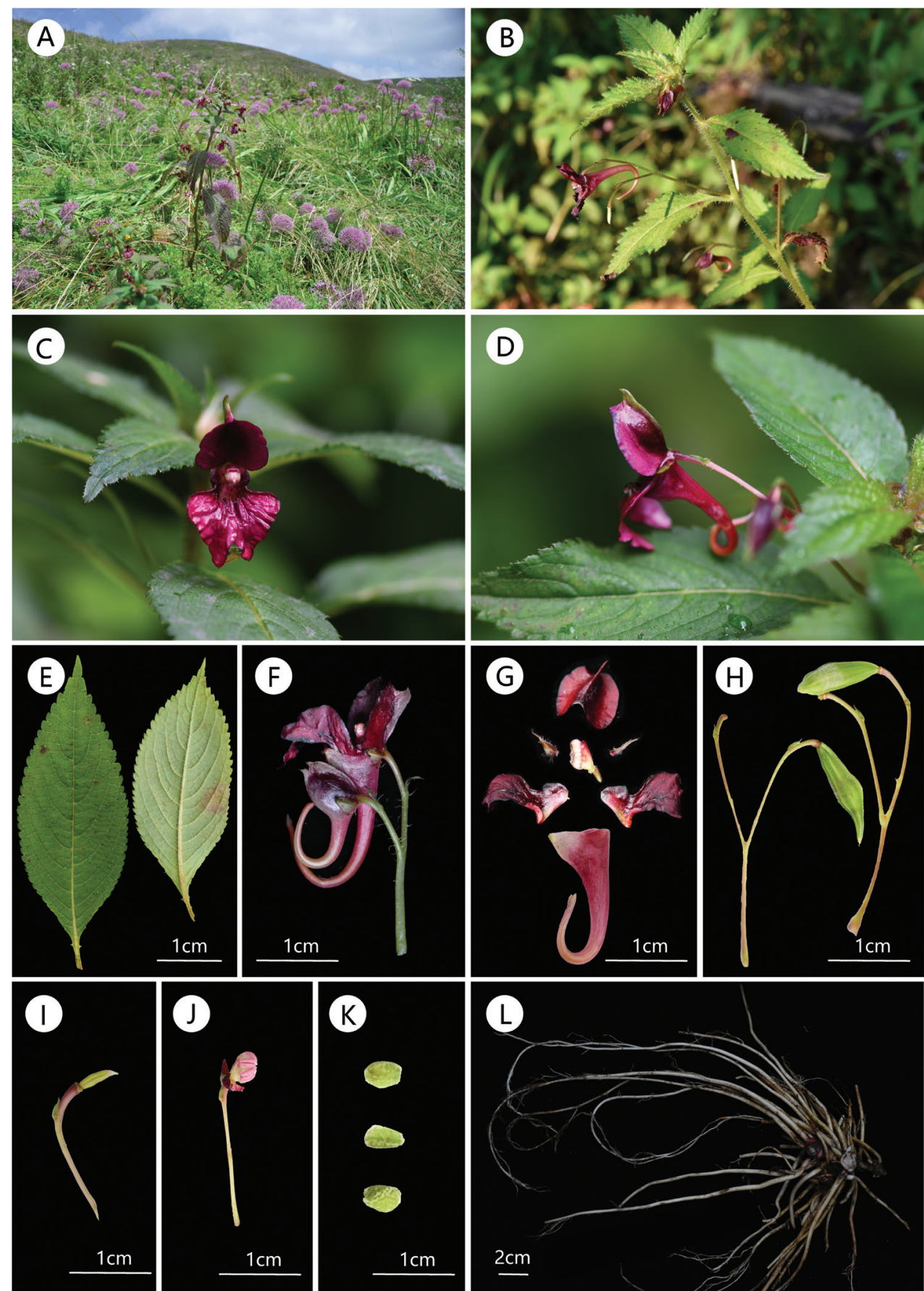

Figure I. Impatiens bijieensis $\mathbf{A}$ habit $\mathbf{B}$ plant $\mathbf{C}$ flower in face view $\mathbf{D}$ flower in lateral view $\mathbf{E}$ leaf adaxial surface (left) and abaxial surface (right) $\mathbf{F}$ inflorescence $\mathbf{G}$ flower dissected $\mathbf{H}$ fruit $\mathbf{I}$ ovary $\mathbf{J}$ anther $\mathbf{K}$ seeds $\mathbf{L}$ root. Photos by Xin-Xiang Bai. 


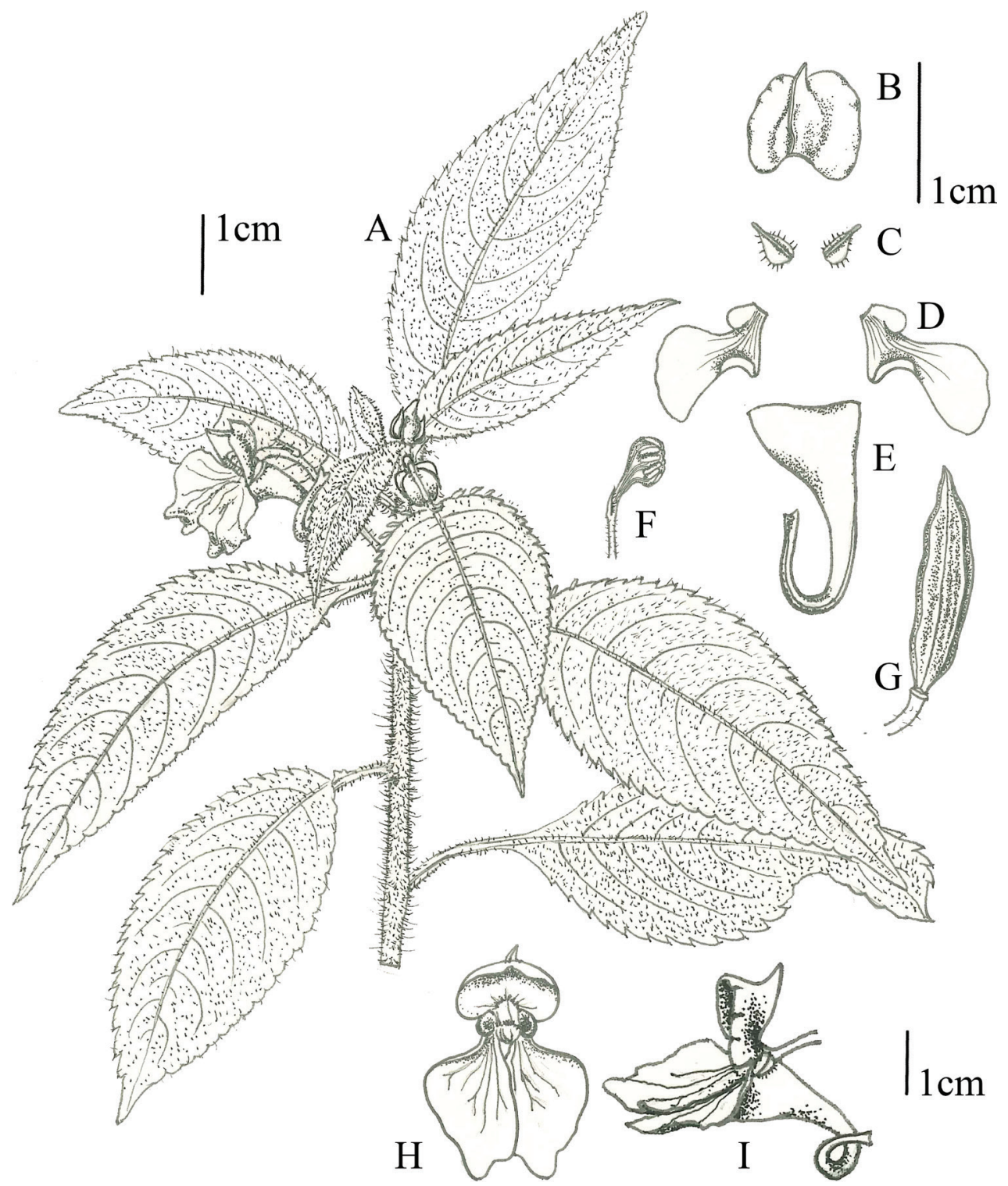

Figure 2. Impatiens bijieensis $\mathbf{A}$ habit $\mathbf{B}$ dorsal petal $\mathbf{C}$ lateral sepal $\mathbf{D}$ lateral united petal $\mathbf{E}$ lower sepal $\mathbf{F}$ anther $\mathbf{G}$ fruit $\mathbf{H}$ flower in face view I flower in lateral view. Drawings by Yi Chen, based on holotype specimen.

Diagnosis. Impatiens bijieensis X.X. Bai \& L.Y. Ren, sp. nov. is similar to I. pterosepala, I. lasiophyton and I. leptocaulon in plant height, leaf blade shape and flower shape, especially resembling I. lasiophyton in its pilosity and obtuse anther apices, but distinguished by its deep purplish-red flower, linear-lanceolate bract, apex 2-lobed lower sepal and cylindrical capsule.

Description. Perennial herb, 30-60 cm high, densely pilose. Root-system developed, with adventitious roots on lower stem nodes. Stem fleshy, erect, branched. 

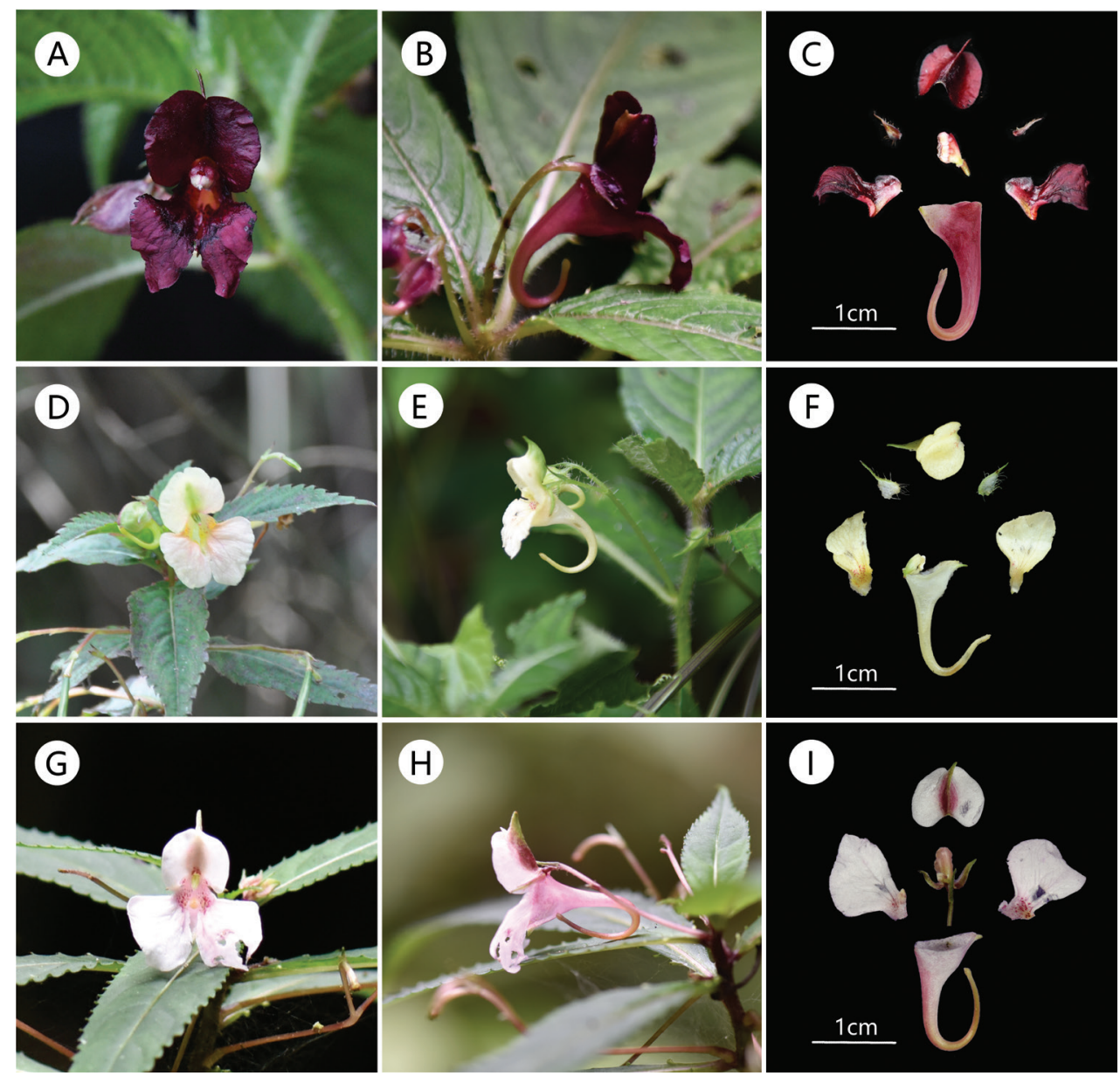

Figure 3. Impatiens bijieensis (A-C) A flower in face view B flower in lateral view $\mathbf{C}$ flower dissected (Photos by X.X. Bai, in Hezhang County, 20 August 2021). Impatiens lasiophyton (D-F) D flower in face view $\mathbf{E}$ flower in lateral view $\mathbf{F}$ flower dissected (Photos by X.X. Bai, in Duyun City, 8 July 2021). Impatiens leptocaulon $(\mathbf{G}-\mathbf{I}) \mathbf{G}$ flower in face view $\mathbf{H}$ flower in lateral view $\mathbf{I}$ flower dissected (Photos by X.X. Bai, in Guiyang City, 22 June 2019).

Leaves simple, alternate, aggregated at stem apex; petiole $0.3-0.8 \mathrm{~cm}$ long; leaf blade elliptic, ovate or ovate-lanceolate, $3.5-5 \mathrm{~cm} \times 1.3-1.8 \mathrm{~cm}$, base cuneate, with a pair of conical glands, margin serrulate, apex acuminate, adaxial surface densely pilose, green; abaxial surface pilose along veins, pale green, lateral veins 6-8 pairs. Inflorescences axillary, 2- or 3-flowered. Peduncles $1.5-1.8 \mathrm{~cm}$ long, pedicels $0.4-0.8 \mathrm{~cm}$ long, slender, bracteate above middle; bract 1 , persistent, linear-lanceolate, $0.3-0.5 \mathrm{~cm}$ long, abaxial mid-vein thickened, margin membranous, ciliate.

Flowers deep purplish-red, 2.3-2.5 cm long. Lateral sepals 2, ovate, ca. $0.5 \mathrm{~cm}$ $\times 0.2 \mathrm{~cm}$, margin ciliate, mid-vein carinate, apex acuminate or caudate. Lower sepal navicular, ca. $0.5 \mathrm{~cm}$ deep (excluding spur), mouth vertical, ca. $0.8 \mathrm{~cm}$ wide, narrowed 
into an incurved, long spur, ca. $2 \mathrm{~cm}$, apex 2-lobed. Dorsal petal reniform, ca. $0.8 \mathrm{~cm}$ $\times 1.5 \mathrm{~cm}$, base truncate or suborbicular, apex concave, abaxial mid-vein carinate, apex acuminate. Lateral united petals sessile, ca. $1.7 \mathrm{~cm}$ long, deep purplish-red with whitish-pink base and reddish patches near auricle, 2-lobed; basal lobes ca. $0.4 \mathrm{~cm} \times 0.2$ $\mathrm{cm}$, oblong to obovate-oblong; distal lobes ca. $1 \mathrm{~cm} \times 0.6 \mathrm{~cm}$, dolabriform. Stamens 5 , filaments linear, anther apices obtuse; ovary 5-carpellate, narrowly fusiform, 0.4-0.5 $\mathrm{cm}$, erect. Capsule fusiform, 1.3-1.8 cm long, apex acuminate, 5-valved, fleshy. Seed ellipsoid, surface with irregular protrusions.

Etymology. The specific epithet 'bijieensis' refers to the type locality where the new species was found, located in Bijie City, northwest Guizhou Province, China. The Chinese name is given as “毕节凤仙花”.

Phenology. Flowers and fruits from August to October.

Distribution. Impatiens bijieensis has been observed in Bijie City, Guizhou Province with subpopulations in Hezhang County and Dafang County. We found at Dajiucaiping, Hezhang that the plants grow along the creek on the slopes of gentle hilltops or on the side slope of the ridge, while some were found below the cliffs where water was dripping from streams. At Jiulongshan, Dafang, the population was distributed in evergreen broad-leaved forest margins.

Ecology. Impatiens bijieensis was collected at elevations of 1915-2800 m. Species of Rubus coreanus Miq. (Rosaceae), Elatostema involucratum Franch. et Sav. (Urticaceae) and Allium wallichii Kunth (Liliaceae) were found to grow in the vicinity of this species.

Conservation status. This species is currently known only from Bijie City, Guizhou Province, China with two subpopulations. The Extent of Occurrence (EOO) is less than $100 \mathrm{~km}^{2}$ and the known Area of Occupancy (AOO) is less than $15 \mathrm{~km}^{2}$. The conservation status can be evaluated as Vulnerable (VU) D2, based on the IUCN Red List Categories and Criteria (IUCN 2019). About 150 and 300 individuals were known in the two subpopulations of Hezhang County and Dafang County, both of which are exposed to human disturbance.

Additional specimen examined. China. Guizhou: Bijie City: Dafang County, Jiulongshan, 27²19'37"N, 105'52'50"E, 1915 m alt., 8 Sep 2021, X.X. Bai et al., JLS 20210908 (GZAC!).

\section{Discussion}

Impatiens bijieensis is similar to I. pterosepala in floral morphology; both have dolabriform distal lobes of lateral united petals and ovate lateral sepals. The former differs from the latter in being pilose (vs. glabrate), having 2-3-flowered (vs. 1-flowered) inflorescences, deep purplish-red (vs. pale purple or purple-red) flower colour, serrulate (vs. crenate) leaf blade margin, conical (vs. globose) glands at leaf bases, mid-veins of dorsal petals carinate (vs. thickened, entire or undulate), margin of lateral sepals ciliate (vs. sometimes denticulate at one side), abaxial mid-veins of lateral sepals carinate (vs. narrowly carinate), anther apices obtuse (vs. acute) and capsules cylindrical (vs. linear). 
Impatiens bijieensis is similar to I. lasiophyton mostly in its pilosity and elliptic, ovate or ovate-lanceolate leaf blades, but it can be easily distinguished from the latter by its deep purplish-red (vs. yellow or white) flowers, thickened abaxial mid-vein and ciliate margin, membranous bract (vs. hirsute bract and inconspicuous mid-vein), navicular and apically 2-lobed lower sepal (vs. broadly funnelform and unlobed), apex concave, abaxial midvein carinate dorsal petal (vs. apex obtuse, abaxial mid-vein thickened, cristate apically), margin of lateral sepal ciliate (vs. lateral sepal hirsute), and cylindrical (vs. linear) capsules.

Impatiens bijieensis is also similar to I. leptocaulon in the length of its petiole, serrulate leaf blade margin and navicular lower sepal, but differs from its adaxial surface densely pilose and abaxial surface pilose along veins (vs. glabrous), ciliate margined lateral sepals and carinate mid-vein (vs. hyaline margined, denticulate on one side). In order to illustrate the morphological circumscription of this new species, we compare the new species with three species with similar morphological characters in Table 1:

Table I. Comparison of morphological characters in Impatiens bijieensis, I. pterosepala (data from Hooker 1910), I. lasiophyton (data from Hooker 1908b) and I. leptocaulon (data from Hooker 1908c).

\begin{tabular}{|c|c|c|c|c|}
\hline Character & I. bijieensis & I. pterosepala & I. lasiophyton & I. leptocaulon \\
\hline $\begin{array}{l}\text { Length of } \\
\text { petiole }\end{array}$ & $0.3-0.8 \mathrm{~cm}$ & $1.5-2.0 \mathrm{~cm}$ & $1-3 \mathrm{~cm}$ & $0.5-1.5 \mathrm{~cm}$ \\
\hline Leaf blade & $\begin{array}{l}\text { with a pair of conical } \\
\text { glands at base, base } \\
\text { cuneate, margin } \\
\text { serrulate, adaxial surface } \\
\text { densely pilose, abaxial } \\
\text { surface pilose along } \\
\text { veins }\end{array}$ & $\begin{array}{l}\text { with } 2 \text { globose basal } \\
\text { glands, base cuneate, } \\
\text { margin crenate, both } \\
\text { surfaces glabrous }\end{array}$ & $\begin{array}{l}\text { base acute, margin } \\
\text { coarsely crenate or } \\
\text { crenate-serrate, both } \\
\text { surfaces hirsute }\end{array}$ & $\begin{array}{l}\text { with few basal glands, } \\
\text { base narrowly cuneate, } \\
\text { margin serrulate, both } \\
\text { surfaces glabrous }\end{array}$ \\
\hline Bract & $\begin{array}{c}\text { bracteate above middle, } \\
\text { linear-lanceolate }\end{array}$ & $\begin{array}{c}\text { bract above middle; } \\
\text { lanceolate }\end{array}$ & $\begin{array}{c}\text { bracteate below flower; } \\
\text { lanceolate }\end{array}$ & $\begin{array}{c}\text { bracteate above middle; } \\
\text { lanceolate }\end{array}$ \\
\hline Flower & deep purplish-red & $\begin{array}{c}\text { pale purple or purple- } \\
\text { red }\end{array}$ & yellow or white & purple-red \\
\hline Lateral sepal & $\begin{array}{c}\text { 2, ovate, margin ciliate, } \\
\text { mid-vein carinate, apex } \\
\text { acuminate or caudate }\end{array}$ & $\begin{array}{c}\text { 2, ovate, margin } \\
\text { sometimes denticulate } \\
\text { at one side, abaxial mid- } \\
\text { vein narrowly carinate, } \\
\text { apex acuminate }\end{array}$ & $\begin{array}{c}2 \text { (or 4), subovate, } \\
\text { hirsute, apex cuspidate }\end{array}$ & $\begin{array}{l}\text { 2, subovate, long } \\
\text { cuspidate, inequilateral, } \\
\text { hyaline margined, } \\
\text { denticulate on one side }\end{array}$ \\
\hline Lower sepal & $\begin{array}{c}\text { navicular, narrowed into } \\
\text { an incurved, long spur, } \\
\text { spur apex 2-lobed }\end{array}$ & $\begin{array}{c}\text { narrowly funnelform, } \\
\text { narrowed into an } \\
\text { incurved, slender spur }\end{array}$ & $\begin{array}{l}\text { broadly funnelform, } \\
\text { gradually narrowed into } \\
\text { an incurved spur }\end{array}$ & $\begin{array}{l}\text { navicular, narrowed into } \\
\text { an incurved, long spur }\end{array}$ \\
\hline Dorsal petal & $\begin{array}{l}\text { reniform, base truncate } \\
\text { or suborbicular, apex } \\
\text { concave, abaxial mid- } \\
\text { vein carinate }\end{array}$ & $\begin{array}{l}\text { orbicular, base cordate, } \\
\text { apex slightly emarginate, } \\
\text { shortly rostellate, abaxial } \\
\text { mid-vein thickened, } \\
\text { entire or undulate }\end{array}$ & $\begin{array}{l}\text { orbicular, base cordate, } \\
\text { apex obtuse, abaxial } \\
\text { mid-vein thickened, } \\
\text { cristate apically }\end{array}$ & $\begin{array}{c}\text { orbicular, base } \\
\text { unknown, apex } \\
\text { rostellate, abaxial mid- } \\
\text { vein carinate }\end{array}$ \\
\hline Basal lobes & $\begin{array}{l}\text { oblong to obovate- } \\
\text { oblong }\end{array}$ & oblong & small or rudimentary & orbicular, small \\
\hline Distal lobes & dolabriform & $\begin{array}{c}\text { broadly dolabriform, } \\
\text { larger }\end{array}$ & $\begin{array}{c}\text { broadly dolabriform or } \\
\text { sublunar }\end{array}$ & obovate-oblong \\
\hline Anther apex & obtuse & acute & obtuse & obtuse \\
\hline Capsule & cylindrical & linear & linear & linear \\
\hline Elevation & $1915-2800 \mathrm{~m}$ & $1500-1700 \mathrm{~m}$ & $1700-2700 \mathrm{~m}$ & $1200-2000 \mathrm{~m}$ \\
\hline
\end{tabular}


Impatiens pterosepala Hook.f. (1910:274), I. lasiophyton Hook.f. (1908b: t. 2871) and I. leptocaulon Hook.f. (1908c: t. 2872). Colour photographs of I. bijieensis, I. lasiophyton and I. leptocaulon are given in Fig. 3 and all of them were taken in Guizhou Province.

The morphological characters, including perennial habit, racemose inflorescence, 5-carpellate ovary, cylindrical capsule and ellipsoidal seeds, indicate that Impatiens bijieensis is a member of the I. subg. Impatiens. It is known that Impatiens mainly grow in places with high relative temperature and low elevations, yet this new species is found in a karst plateau area that is characterised by its cold climate and high altitude. These ecological characters distinguish it from morphologically-similar species. Additionally, because of the development of tourism, its habitat is currently threatened by human activities.

\section{Acknowledgement}

This research was supported by National Natural Science Foundation of China (31960328) and Survey of Wild Ornamental Plant Resources in Guizhou Province (701256192201).

\section{References}

Cai XZ, Hu GW, Cong YY (2015) Impatiens xanthinoides (Balsaminaceae), a new species from Yunnan, China. Phytotaxa 227(3): 261-267. https://doi.org/10.11646/phytotaxa.227.3.5 Chen YL (2001) Balsaminaceae. In: Chen YL (Ed.) Flora Reipublicae Popularis Sinica (Vol. 47(2)), Sapindales. Science Press, Beijing, 1-243.

Chen YL, Akiyama S, Ohba H (2007) Balsaminaceae. In: Wu ZY, Raven PH, Hong DY (Eds) Flora of China (Vol. 12). Science Press, Beijing \& Missouri Botanical Garden Press, St. Louis, 43-113.

Cong YY (2010) Study on the flora and phylogeny of Impatiens L. in Hengduan Mountains. $\mathrm{PhD}$ Thesis, Hunan Normal University, Changsha.

Ding B, Gadagkar SR, Wang JC, Zhang M, Guo H, Yu SX (2016) Impatiens wawuensis (Balsaminaceae): A new species from Sichuan, China. Phytotaxa 273(4): 293-298. https:// doi.org/10.11646/phytotaxa.273.4.5

Ding B, Wang JC, Deng HP, Wang CY (2017) Impatiens baishaensis (Balsaminaceae): A new species from Sichuan, China. Phytotaxa 319(2): 191-196. https://doi.org/10.11646/ phytotaxa.319.2.8

Grey-Wilson C (1980) Impatiens of Africa. CRC Press, Rotterdam, 235 pp.

Gu JZ, Yang N, Peng YC, Song YX, Guo SW, Cong YY (2021) Impatiens shangjiangensis (Balsaminaceae), a new species from Yunnan, China. Annales Botanici Fennici 58(4-6): 197-201. https://doi.org/10.5735/085.058.0401

Hooker JD (1908a) Les espèces du genre "Impatiens" dans l'Herbier du Muséum de Paris.

Nouvelles Archives du Muséum d'Histoire Naturelle, Paris, sér. 4 10: 233-272.

Hooker JD (1908b) Impatiens lasiophyton, Hk.f. Hooker's Icones Plantarum 29: t.2871.

Hooker JD (1908c) Impatiens leptocaulon, Hk.f. Hooker's Icones Plantarum 29: t.2872. 
Hooker JD (1910) XXXVIII, - New Impatiens from China. Bulletin of Miscellaneous Information (Royal Botanic Gardens, Kew) 1910: 269-274. http://www.jstor.org/stable/4111718

IUCN (2019) Guidelines for using the IUCN Red List Categories and Criteria. Version 14. Prepared by the Standards and Petitions Subcommittee, 116 pp. http://www.iucnredlist. org/documents/RedListGuidelines.pdf

Kuang RP (2015) Study on the flora in Guizhou/southern Sichuan and phylogenetic problems of Impatiens L. PhD Thesis, Hunan Normal University, Changsha.

Kuang RP, Duan LD, Gu JZ, Cai XZ, Cong YY, Liu KM (2014) Impatiens liboensis sp. nov. (Balsaminaceae) from Guizhou, China. Nordic Journal of Botany 32(4): 463-467. https:// doi.org/10.1111/j.1756-1051.2013.00194.x

Liao RL, Cai L, Yu ZY, Wang YH, Sun WB (2021) Impatiens wutaishanensis (Balsaminaceae), a new species from Southeast Yunnan, China. PhytoKeys 176: 43-53. https://doi. org/10.3897/phytokeys.176.58825

Linnaeus C (1753) Species Plantarum 2. Laurentius salvius, Stockholm, 937 pp.

Luo Y, Deng LX [Eds] (2015) Impatiens. Guizhou Vascular Plant Catalogue. China Forestry Publishing House, Beijing, 342-344.

Mabberley DJ (2017) Mabberley's Plant-book, a portable dictionary of plants, their classification and uses. Cambridge University Press, 1040 pp. https://doi.org/10.1017/9781316335581

Peng S, Cong YY, Tian J, Zhang CF, Hu GW, Wang QF (2021a) Impatiens bullatisepala (Balsaminaceae), a new species from Guizhou, China. Phytotaxa 500(3): 217-224. https:// doi.org/10.11646/phytotaxa.500.3.5

Peng S, Rono CP, Yang JX, Wang JJ, Hu GW, Wang QF (2021b) Description of a new species and lectotypification of two names in Impatiens Sect. Racemosae (Balsaminaceae) from China. Plants 10(9): 1-11. https://doi.org/10.3390/plants10091812

Song Y, Yuan Y-M, Küpfer P (2003) Chromosomal evolution in Balsaminaceae, with cytological observations on 45 species from Southeast Asia. Caryologia 56(4): 463-481. https://doi.or g/10.1080/00087114.2003.10589359

Song YX, Cong YY, Kuang RP, Peng YC, Zheng YM (2021a) Impatiens sterilis, a rare new species of Impatiens with reduced sterile inflorescences from Yunnan, China. Taiwania 66(1): 48-52. https://doi.org/10.6165/TAI.2021.66.48

Song YX, Peng S, Cong YY, Zheng YM (2021b) Impatiens rapiformis, a new species of Impatiens with root tuber from Yunnan, China. Nordic Journal of Botany 39(5): njb.03151. https:// doi.org/10.1111/njb.03151

Song YX, Xiao Y, Peng S, Cong YY, Hu GW (2021c) Two new species of Impatiens from China, and taxonomic insights into the Longiflamenta Group, which is endemic to China. Plants 10(8): 1-15. https://doi.org/10.3390/plants10081697

Tan YH, Liu YN, Jiang H, Zhu XX, Zhang W, Yu SX (2015) Impatiens pandurata (Balsaminaceae), a new species from Yunnan, China. Botanical Studies 56: e29. https:// doi.org/10.1186/s40529-015-0108-4

Xia CY, Sudhindra RG, Zhao XL, Do TV, Zhu XY, Qin Y, Yu SX (2019) Impatiens maculifera sp. nov. (Balsaminaceae) Yunnan, China. Nordic Journal of Botany 37(8): e02422. https:// doi.org/10.1111/njb.02422

Xiong YX, Luo YC (1989). Balsaminaceae. In: Li YK (Ed.) Flora of Guizhou, Vol. 7. Sichuan National Press, Chengdu, 626-641. 
Xiong YX, Yang CD (2009) Illustrated handbook of common herbaceous seed plants in Fanjing Mountain National Nature Reserve. Guizhou Science and Technology Publishing House Co., Ltd, Guiyang, 91-95.

Yu SX (2012) Balsaminaceae of China. Peking University Press, Beijing, 214 pp.

Yu S, Li MJ, Yuan TH, Ren LY, Bai XX (2021) Species diversity and geographic distribution of wild Impatiens in Guizhou Province. Xibei Zhiwu Xuebao 41: 863-871.

Yuan YM, Song Y, Geuten K, Rahelivololona E, Wohlhauser S, Fischer E, Smets E, Küpfer P (2004) Phylogeny and biogeography of Balsaminaceae inferred from ITS sequences. Taxon 53(2): 391-403. https://doi.org/10.2307/4135617

Yuan TH, Li MJ, Yu S, Ren LY, Huang RX, Chen Y, Bai XX (in press) Impatiens liupanshuiensis (Balsaminaceae), a new species from Guizhou, China. PhytoKeys. 